\title{
Detección precoz del cáncer de laringe en Cuba
}

\author{
Early detection of laryngeal cancer in Cuba
}

\section{Juan J . Lence Anta'; Leticia M. Fernández Garrote"}

'Especialista de II Grado en Bioestadística. Investigador Auxiliar. Instituto Nacional de Oncología y Radiobiología. La Habana, Cuba.

"Doctora en Ciencias Médicas. Investigadora Titular. Escuela Nacional de Salud Pública. La Habana, Cuba.

\section{RESUMEN}

I ntroducción La laringe constituye un órgano anatómicamente complejo. Glotis, supraglotis y subglotis son, en orden de importancia relativa, los sitios más frecuentes de asentamiento de tumores, los cuales se distinguen clínicamente por el modo en que afectan las principales funciones de la laringe: fonación y deglución. Objetivos Describir la frecuencia, características sociodemográficas y tumorales correspondientes a los casos incidentes de cáncer de laringe en Cuba según subsitio anatómico dentro de la laringe.

Métodos Se realizó un estudio descriptivo a partir de los casos nuevos de cáncer de laringe reportados al Registro Nacional de Cáncer durante el período 1988-2003. Se analizó información sociodemográfica de los casos y otras variables relacionadas con el diagnóstico y la enfermedad tumoral. Se utilizó la prueba Chi-cuadrado de independencia para determinar asociación entre las variables en estudio con un nivel de significación de 0,05.

Resultados El cáncer de laringe ocupa una de las primeras causas de incidencia y mortalidad por cáncer en Cuba, fundamentalmente en hombres entre 50-74 años de edad. Mientras que el cáncer de subglotis es poco frecuente, el cáncer de glotis es hasta tres veces más frecuente que el de supraglotis.

Conclusiones El cáncer de glotis da síntomas tempranamente pudiendo ser detectado mediante un examen relativamente sencillo. Considerando las 
características del sistema nacional de salud cubano, este hecho puede abrir una expectativa al diagnóstico temprano, por lo que es recomendable evaluar la factibilidad y eficacia del examen otorrinolaringológico -como parte del examen periódico anual- fundamentalmente en hombres de 50 años y más, con historia de tabaquismo o alcoholismo, con el fin de detectar y tratar tempranamente la enfermedad.

Palabras clave: Cáncer de laringe, incidencia, prevención secundaria, glotis, supraglotis, laringoscopia indirecta, Cuba.

\section{ABSTRACT}

Introduction Larynx is an anatomically complex organ. Glottis, supraglottis and subglottis are, in order of relative importance, the most frequent tumor locations, which are clinically characterized by the way they affect the main functions of the larynx: phonation and deglutition.

Objectives the present paper describes the frequency, socio-demographic and tumorous characteristics of laryngeal cancer cases in Cuba.

Methods A descriptive study was conducted, taking the new laryngeal cancer cases reported to the National Register of Cancer from 1988 to 2003 as a basis. Social and demographic information of cases and other variables related to diagnosis and to the tumorous disease were analyzed. Chi-square test of independence determined the association of variables under study, with significance level equal to0,05.

Results Laryngeal cancer is one of the first causes of incidence and mortality due to cancer in Cuba, mainly in males aged $50-74$ years. Although subglottis cancer is rare, glottis cancer is up to three times as frequent as that of supraglottis.

Conclusions Glottis cancer shows early symptoms, so it can be detected on a relatively simple examination. Considering the Cuban health system characteristics, this event could open up possibilities for early detection, therefore, it would be advisable to evaluate the feasibility and efficacy of the otorhinolaryngology test -as part of the annual systematic examination - mainly in men aged 50 years and more, with history of smoking/alcoholism, in order to detect and treat the disease early.

Key words: Larynx cancer, incidence, secondary prevention, glottis, supraglottis, indirect laryngoscopy, Cuba.

\section{NTRODUCCIÓN}

La carcinogénesis del carcinoma de laringe es compleja y hoy día se llevan a cabo múltiples estudios etiológicos para determinar el papel de los genes y virus en el desarrollo del cáncer; sin embargo, aún cuando se han encontrado receptores androgénicos en el tejido tumoral laríngeo y se han descrito casos de cáncer de laringe en la mujer, con debut durante el primer embarazo y con recidivas en cada una de las siguientes gestaciones, ${ }^{1}$ así como algunos factores medioambientales, 
ocupacionales y dietéticos que juegan un papel en la carcinogénesis del carcinoma laríngeo; el alto consumo de tabaco y alcohol generalmente se consideran los principales factores de riesgo del cáncer de laringe. ${ }^{2-5}$

La laringe es un órgano anatómicamente complejo (fig. 1), cuyos límites anatómicos son la orofaringe, que la limita anteriormente, y la hipofaringe que la circunda posteriormente. La epilaringe es la unión entre el sistema respiratorio y el tracto digestivo. ${ }^{6}$ La endolaringe se divide en tres regiones anatómicas y funcionales: supraglotis, glotis y subglotis y agrupa en general los principales subsitios de asentamiento de tumores. Las diferentes versiones de la Clasificación Internacional de Enfermedades para Oncología, no distinguen con exactitud estas divisiones anátomo-funcionales, considerando algunos tumores de la epilaringe dentro de los tumores supraglóticos. ${ }^{6}$

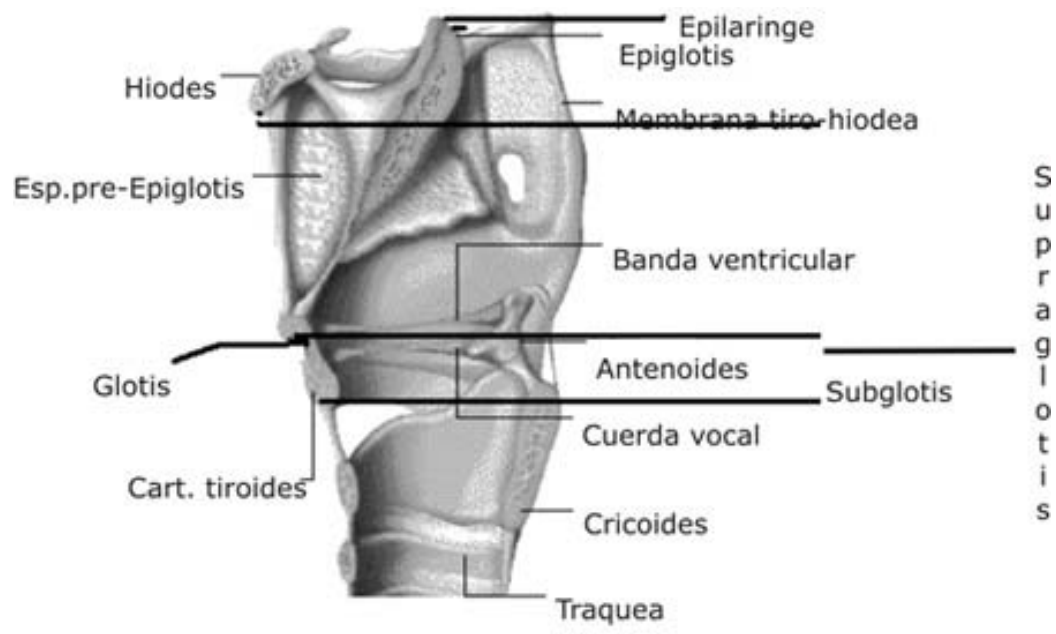

Fuente: Ob. cit.7.

Fig. 1. Regiones en que se divide la laringe.

Los tumores subglóticos son raros, comprenden solamente un escaso porcentaje de tumores malignos de la laringe. ${ }^{1,7,8}$ Algunos tumores de más rara incidencia, llamados marginales, incluyen los tumores que afectan los márgenes de la laringe, o sea los repliegues aritenoepiglóticos o faringoepiglóticos, los senos piriformes, la cara lingual de la epiglotis o la base de la lengua. ${ }^{1}$ La frecuencia de tumores glóticos comparados con lesiones supraglóticas varía en diferentes partes del mundo. ${ }^{9-13}$ Los primeros predominan generalmente sobre los segundos, con una razón glotis: supraglotis de aproximadamente $2: 1$.

Algunos cánceres del límite superior de la laringe (epilaringe) no son siempre claramente distinguidos de aquellos de la hipofaringe, por lo que algunos estudios sobre la epidemiología del cáncer de laringe incorporan también los tumores de hipofaringe. En este sentido se ha descrito que la hipofaringe y la epilaringe están más expuestas a sustancias ingeridas mientras que la endolaringe lo está a carcinógenos inhalados. ${ }^{6}$

Aunque el diagnóstico de certeza lo proporciona la anatomía patológica, sólo con la anamnesis y la exploración física es posible identificar la inmensa mayoría de los cánceres de laringe. La sintomatología varía según la localización del tumor, su tamaño, el estado general del enfermo y otros, pero existen una serie de síntomas mayores ocasionados por la interferencia que la masa tumoral origina en las 
funciones laríngeas: disfagia u odinofagia, disfonía y disnea. ${ }^{1}$ Ya que la laringe supraglótica participa en las funciones deglutorias, la presencia de un tumor supraglótico origina frecuentemente disfagia u odinofagia. Los pacientes refieren sensación de cuerpo extraño faríngeo, carraspeo y molestias inespecíficas al tomar irritantes, como cítricos o licores. La invasión de la zona marginal lateral o del piriforme ocasiona frecuentemente otalgia refleja. La disfonía es infrecuente, pero cuando el tumor crece puede aparecer una voz engolada sin que se modifique la frecuencia fundamental de la voz. Dada la pobre sintomatología que provocan estos tumores, no es raro que el motivo de consulta sea la aparición de una adenopatía metastásica cervical. ${ }^{1}$

Los tumores glóticos se diagnostican más precozmente ya que producen disfonía persistente y progresiva, ocasionada por la interferencia de la masa con el cierre de la glotis. Cuando el tumor crece e invade el músculo tiroaritenoideo la cuerda se paraliza y aumenta la gravedad de la disfonía. La fijación de la cuerda vocal ocasiona incompetencia glótica y pueden aparecer aspiraciones, y si el tumor llega a obstruir una porción importante del espacio glótico puede provocar disnea. Por su parte los tumores subglóticos son clínicamente silentes y sólo producen disfonía cuando alcanzan la glotis, pero es frecuente que produzcan disnea y estridor. Otros síntomas más tardíos pueden ser: tos, hemoptisis, halitosis, astenia, anorexia y pérdida de peso. ${ }^{1}$

La exploración de la laringe se basa en cinco puntos fundamentales: inspección, valoración de la voz, palpación laríngea, palpación cervical y laringoscopia indirecta. ${ }^{14}$ La laringoscopia directa y los métodos de imagen se utilizan para la valoración de la extensión del tumor. La laringoscopia directa es un método más invasivo y más adecuado para la confirmación diagnóstica.

Cuba se encuentra entre los países con más alta morbilidad y mortalidad por cáncer de laringe en ambos sexos, ocupando el cuarto lugar en orden de importancia relativa en hombres -alrededor del $6 \%$ del total de todos los casos nuevos de cáncer reportados en hombres- excluyendo piel. ${ }^{15}$

Teniendo en cuenta los aspectos diferenciales atribuidos a los diferentes subsitios anatómicos del cáncer de laringe, el presente trabajo tiene por objetivo describir la frecuencia y características sociodemográficas y tumorales correspondientes a los casos incidentes reportados al Registro Nacional de Cáncer (RNC), durante el periodo 1988-2003, según subsitio anatómico dentro de la laringe. Un mejor conocimiento de estos aspectos podría facilitar el establecimiento de políticas y estrategias encaminadas a la prevención de la enfermedad.

\section{MéTODOS}

Se realizó un estudio descriptivo tomando como universo de estudio los casos nuevos de cáncer de laringe -Clasificación Internacional de Enfermedades para Oncología (CIEO-1): ${ }^{16}$ códigos 161.0-161.9- reportados al Registro Nacional de Cáncer (RNC), durante el período 1988-2003. A partir de las bases de datos del registro se tomó y analizó la información sociodemográfica de los casos (edad, sexo) y otras variables relacionadas con el diagnóstico y la enfermedad tumoral, tales como: subsitio anatómico y etapa clínica al diagnóstico. Se utilizó la prueba Chi-cuadrado de independencia para determinar asociación entre las variables en estudio con un nivel de significación de 0,05. 


\section{RESULTADOS}

Entre 1988 y el año 2003 se reportaron al RNC un total de 12899 casos nuevos de carcinoma de la laringe (CIEO-1: 161.0-161.9), aproximadamente 800 casos anuales, de los cuales el $84 \%$ fueron hombres - a razón de 5 y 6 hombres por cada mujer, la razón hombre: mujer parece tener un comportamiento bastante estable a través de la serie.

Aun cuando en el $76 \%$ de los casos nuevos informados al RNC, la topografía no pudo ser especificada (CIEO-1: 161.9), o no fue completada, se observó en general una mayor frecuencia de la localización glótica con una razón global glotis:

supraglotis de 3 , mientras que la localización subglótica fue generalmente rara ( $1 \%$ de los casos). La frecuencia relativa del cáncer de la glotis fue significativamente superior en hombres (tabla 1$)$, que en mujeres $(p<0,01)$. La razón glotis: supraglotis fue dos veces mayor en hombres $(3,4)$ que en mujeres $(1,5)$.

Tabla 1. Distribución de los casos según sexo y sitio topográfico dentro de la laringe.

Cuba (1988-2003)

\begin{tabular}{|c|c|c|c|c|c|}
\hline Topografia* & $\begin{array}{c}\text { Glotis } \\
\text { CIE } 161.0\end{array}$ & $\begin{array}{l}\text { Subglotis } \\
\text { CIE } 161.2\end{array}$ & $\begin{array}{l}\text { Supraglotis } \\
\text { CIE } 161.1\end{array}$ & Total & Razón** \\
\hline Sexo & No. (\%) & No. (\%) & No. (\%) & No. & \\
\hline Hombres & $2002(76)$ & $30(1)$ & $582(22)$ & 2614 & 3,4 \\
\hline Mujeres & $269(58)$ & $8(2)$ & $183(40)$ & 460 & 1,5 \\
\hline Total & $2271(74)$ & 38 (1) & $765(25)$ & 3074 & 3 \\
\hline
\end{tabular}

$x^{2}(2 g l)=66,7 \quad(p=0,000)$.

* Se excluyeron 9797 casos con localización no definida +28 casos de cartílago laríngeo (CIEO-1: 161.3), ** Razón glotis: supraglotis.

La distribución relativa del tumor de glotis en relación con el supraglótico es consistente a través de los años estudiados. El cáncer glótico oscila entre el 73 y el $76 \%$.

La edad promedio de los casos era de 65,2 años (IC $95 \%$ : 65, 0-65,4). El $70 \%$ (2 $134 / 3$ 054) de los casos se diagnosticó entre los 50 y 74 años. Aun cuando la distribución de los subsitios topográficos en los diferentes grupos de edad mostró un patrón bastante homogéneo -con una frecuencia entre 71 y $79 \%$ para el cáncer de glotis-, un porcentaje relativamente más alto de tumores glóticos se observó en los grupos más jóvenes - por debajo de los 64 años (tabla 2). 
Tabla 2. Distribución de los casos según la edad al diagnóstico en los principales sitios topográficos dentro de la laringe. Cuba (1988-2003)

\begin{tabular}{|c|c|c|c|c|}
\hline Topografía* & Glotis & Subglotis & Supraglotis & Total \\
\hline Edad** & No. (\%) & No. (\%) & No. (\%) & No. \\
\hline$<50$ & $294(79)$ & $7(2)$ & 70 (19) & 371 \\
\hline $50-64$ & $951(75)$ & 11 (1) & 305 (24) & 1267 \\
\hline $65-74$ & $619(71)$ & 12 (1) & $236(27)$ & 867 \\
\hline 75 y más & $395(72)$ & $8(1)$ & $146(27)$ & 549 \\
\hline Total & $2259(74)$ & $38(1)$ & 757 (25) & 3054 \\
\hline
\end{tabular}

$x^{2}(6 g l)=16,7 \quad(p=0,01)$.

* Se excluyeron 9797 casos con localización no definida +28 casos de cartílago laríngeo (CIE 161.3), ** En 63 casos la edad no fue definida.

La etapa clínica al momento del diagnóstico era desconocida en aproximadamente el $45 \%$ ( 5 793/12 899) de los casos nuevos de cáncer de laringe informados al RNC, aunque esta proporción ha disminuido durante la última década, de 57 \% ( 1 293/2 273) en el trienio 1994-96 a 33 \% ( 1 203/3 608) en el período 2000-2003. En poco más de la mitad de los casos en que no se notifica el sitio topográfico la etapa clínica es desconocida. Sin embargo si se excluyen los casos en que se desconoce el sitio topográfico, la proporción de casos con etapa desconocida se encuentra alrededor de $23 \%$ con una distribución bastante homogénea entre los diferentes subsitios.

Aunque más de la mitad de los casos con subsitio topográfico definido fueron registrados en etapa I y II, la distribución por etapa clínica al diagnóstico fue significativamente diferente en los dos principales sitios topográficos: un porcentaje significativamente menor $(p<0,000)$ de diagnósticos tardíos (III y IV) se encontró en los casos con tumores glóticos (13\%), en contraste con los casos con tumores supraglóticos donde el $37 \%$ se diagnosticó en estadio avanzado de la enfermedad (tabla 3).

Tabla 3. Distribución de los casos según etapa clínica al diagnóstico en los principales sitios topográficos. Cuba. 1988-2003

\begin{tabular}{|l|c|c|c|c|}
\hline \multicolumn{1}{|c|}{ Topografía } & Glotis & Subglotis & Supraglotis & Total \\
\hline \hline Etapa clínica & No. (\%) & No. (\%) & No. (\%) & No. (\%) \\
\hline I - II & $1457(64)$ & $20(53)$ & $318(42)$ & $1795(58)$ \\
\hline III y IV & $290(13)$ & $8(21)$ & $282(37)$ & $580(19)$ \\
\hline Desconocida & $524(23)$ & $10(26)$ & $165(22)$ & $699(23)$ \\
\hline Total & $2271(100)$ & $38(100)$ & $765(100)$ & $30748(100)$ \\
\hline
\end{tabular}

$$
x^{2}(4 g l)=227,15 \quad(p=0,000) .
$$

* Se excluyeron 9797 casos con localización no definida + 28 casos de cartílago laríngeo (CIE 161.3). 


\section{DISCUSIÓN}

El riesgo de enfermar de cáncer de laringe en Cuba es entre cinco y seis veces más frecuente en hombres que en mujeres. Esta cifra es generalmente baja comparada con otros países de Europa, ${ }^{17}$ donde oscila entre 10 y 30. Esta razón es estimada marcadamente más alta en España: 63 y 49 en Asturias y Zaragoza, ${ }^{18}$ en Italia (Trieste) y Polonia, con valores de 10 y 11 , respectivamente. ${ }^{19}$ Valores superiores a 17 son registrados en el departamento de la Somme (Francia), ${ }^{20}$ entre 1984- 1995. En estos países las tasas en hombres son notablemente más altas, sin embargo, las tasas en mujeres son alrededor de la mitad de las encontradas en Cuba. En este país, en una serie de casos estudiados en Camagüey ${ }^{19}$ entre 1985-1992 la razón hombre: mujer notificada es de 5,5.

En Cuba más del $65 \%$ de las neoplasias malignas ocurren en personas de la tercera edad. ${ }^{21}$ El tumor maligno de la laringe sigue un patrón similar al registrado para otras neoplasias, considerada como una enfermedad fundamentalmente del adulto mayor, con un pico alrededor de los 65 años. La indicación de que el cáncer laríngeo es distintivo de la tercera edad sugiere la necesidad de un pesquisaje más activo por los profesionales médicos de la atención primaria hacia este grupo vulnerable.

La frecuencia del cáncer de glotis en Cuba es superior al de supraglotis, lo cual es consistente con los hallazgos encontrados en estudios de serie de casos en Canadá, Dinamarca, Inglaterra y Los Estados Unidos. ${ }^{22}$ En Cuba, la razón glotis: supraglotis aparece un tanto más elevada que en otros países, y es dos veces mayor en hombres que en mujeres, con una mayor proporción en los menores de 50 años. Se debe tener en cuenta que en el 76 \% de los casos notificados al RNC, la localización no fue definida, lo que de algún modo podría sugerir que la frecuencia relativa de cáncer de la supraglotis podría estar ligeramente subestimada.

Por su parte, el cáncer de la supraglotis ha sido encontrado más prevalente en ciertas regiones de Francia, Italia y España, en este último país, en Murcia, la región supraglótica fue la localización más común (67\%). 6,23-26 Mayor incidencia de cáncer supraglótico ha sido también encontrado en Turquía, ${ }^{27,28}$ en América Latina (Uruguay), ${ }^{29,30}$ y en Finlandia, ${ }^{31}$ donde la razón de incidencia glótico: supraglótico parece haber ido cambiando.

De acuerdo a los estudios basados en reportes de series de pacientes tratados en el Hospital Central de la Universidad de Helsinki entre 1936-61, las dos terceras partes de los cánceres laríngeos fueron supraglóticos. ${ }^{22,32}$ Publicaciones posteriores, que incluyen datos de cinco hospitales universitarios de Finlandia, entre el 50 y 68 $\%$ de los cánceres de la laringe localizados durante 1980 y 1990 fueron glóticos. ${ }^{31}$ Los efectos del alcohol y el tabaco han estado asociados diferencialmente a estos principales subsitios anatómicos. El cáncer de la supraglotis se encuentra más fuertemente asociado al consumo de tabaco y alcohol que el cáncer de glotis. ${ }^{33-35}$

Similar a lo observado en el presente estudio, la frecuencia relativamente mayor de tumores supraglóticos en mujeres que en hombres es registrada como un hallazgo usual. ${ }^{9-12,26,36,37}$

En este estudio las lesiones glóticas son más frecuentes que las supraglóticas para todos los grupos de edad, incluso en los grupos más jóvenes se observó mayor incidencia de lesiones glóticas. En algunos estudios las lesiones supraglóticas han sido más comunes en pacientes más jóvenes. ${ }^{32,12,38}$ 
Los datos muestran que una significativa proporción de pacientes son registrados en etapa clínica desconocida, esta proporción es marcadamente alta en los casos en que no se determina el diagnóstico topográfico. Se ha detectado que la distribución de diagnósticos con etapa clínica desconocida está generalmente sesgada hacia los estadios más avanzados; así lo demuestran estudios de supervivencia en cáncer de mama [Fernández Leticia, Galán Y (J' del RNC). Comunicación personal, 2008]. La falta de estadiamiento de los casos constituye un diagnóstico clínico incompleto y hace suponer un mal manejo terapéutico en estos pacientes.

Aún cuando más del $40 \%$ de los casos se diagnostican en etapas más precoces, esta proporción es significativamente alta en pacientes con tumores glóticos - $64 \%$ vs. $41 \%$ en pacientes con cáncer de la supraglotis. Estos resultados son en general consistentes con los informados por otros autores. Se ha señalado que los pacientes con cáncer supraglótico tienen peor pronóstico debido a la incidencia de tumores histológicamente más agresivos, pero clínicamente silentes, diagnosticándose en etapas más avanzadas, que los pacientes con tumores glóticos, ${ }^{1,39}$ los cuales se hacen sintomáticos más tempranamente. De forma coherente a lo planteado por Hirvikoski, ${ }^{39}$ una frecuencia significativamente mayor de diagnósticos en etapas III y IV se observa en tumores supraglóticos en relación con el tumor de glotis.

Teniendo en cuenta la alta incidencia del cáncer de laringe en Cuba, es una de las cinco causas de cáncer más frecuente en hombres y constituye en orden de importancia relativa el tumor más frecuente de las vías aero-respiratorias superiores, fundamentalmente a partir de la quinta y sexta década de la vida, se subraya en la necesidad de desarrollar estrategias inaplazables para favorecer su prevención y control.

Dentro de las posibilidades que brinda el Sistema Nacional de Salud en Cuba y desde el punto de vista de la prevención primaria, es necesario continuar insistiendo en la cesación por la población de los hábitos de tabaquismo y alcoholismo, principales factores de riesgo de la enfermedad. Por su parte, el cambio del timbre de la voz en sujetos de 50 años y más, con historia de tabaquismo y/o alcoholismo, debe constituir también una señal de alerta y deben, por tanto, ser incorporado a los programas de promoción y control del cáncer. Se afirma que el diagnóstico de cáncer de laringe debe ser altamente sospechado ante todo paciente mayor de 50 años, fumador o bebedor habitual que presente disfonía o molestias faríngeas de más de 15 días de evolución. ${ }^{1}$

La detección temprana del cáncer de laringe ha recibido en general poca atención, tanto por parte de la literatura especializada como de las instituciones internacionales que rigen las políticas de control de cáncer. ${ }^{40}$

Aun cuando en algunas poblaciones la incidencia de cáncer de laringe no justifica la puesta en marcha de un programa de pesquisaje, algunos médicos han recomendado el pesquisaje en pacientes de alto riesgo (fumadores severos). ${ }^{41} \mathrm{La}$ mayoría de los estudios sin embargo, son consistentes en que la ronquera constituye un signo temprano de cáncer de laringe, especialmente de cáncer de glotis, y recomiendan a los médicos chequear a aquellos pacientes que se quejan de ronquera o de cambios en la calidad de la voz y referirlos a un otorrinolaringólogo. La detección de cáncer de laringe en estos casos es entre 3 y $5 \%$.

Probablemente uno de los estudios pioneros que se refieren al diagnóstico temprano del cáncer laringeo es el de T. Hoare y otros, ${ }^{42}$ a principios de la década 
del 90, en el Reino Unido, donde se señalan las ventajas del nasoendoscopio flexible de fibra óptica, que revolucionó la técnica endoscópica para el diagnóstico y tratamiento de la enfermedad laríngea. Aunque en el Reino Unido la incidencia de cáncer de laringe no es alta (alrededor de $1 \%$ de todos los casos registrados en hombres), Hoare y otros determinan la validez del médico general para sospechar el cáncer de laringe y evalúan si la referencia y el acceso inmediato a un centro especializado constituye una forma efectiva de asegurar la detección temprana de la enfermedad. De una serie de 300 pacientes, 232 fueron remitidos al otorrinolaringólogo por ronquera, de ellos 39 casos fueron remitidos para laringoscopia directa y en el $25 \%$ se comprueba tumor maligno por histología (3,3 $\%$ del total).

Abtahi en Irán, ${ }^{43}$ apunta también a la laringoscopia directa como regla de oro para el diagnóstico de cáncer de laringe en pacientes con ronquera persistente. De una serie de 363 pacientes evaluados con ronquera de dos semanas de evolución, en el $20 \%$ se confirma lesión tumoral por histología. Estos representan el $58 \%$ de 131 pacientes con posible diagnóstico de tumor por laringoscopia directa.

Más recientemente ha sido señalado que la laringoscopia autofluorescente directa facilita la detección de lesiones precancerosas, carcinoma in situ y cáncer microinvasivo más exactamente que la observación clínica sola. ${ }^{44}$ En una serie de 127 pacientes sospechosos de presentar lesiones cancerosas y precancerosas de la laringe diagnosticados por microlaringoscopia, la endoscopía autofluorescente fue comparada con los hallazgos histopatológicos, obteniéndose una sensibilidad y especificidad de $97,3 \%$ y $83,8 \%$, respectivamente. Aunque se señala que la cicatrización, la hiperqueratosis y la inflamación pueden limitar el valor predictivo del método.

Por su parte la laringoscopia indirecta por autofluorescencia parece también ser prometedora como herramienta en el diagnóstico temprano del cáncer de laringe y sus lesiones precursoras. Esta ha sido evaluada frente a la laringoscopia directa por autofluorescencia y frente a los hallazgos histopatológicos ${ }^{45}$ y se han encontrado resultados concordantes en el $89 \%$ de los casos.

Olofsson ${ }^{46}$ subraya que los pacientes con trastornos de la voz deben ser examinados preferiblemente por laringoestroboscopía o videolaringoestroboscopía, mientras que pacientes con laringitis crónica, queratosis y eritroplasia deben ser vigilados más de cerca. Dentro de los procedimientos diagnósticos se cita que la microlaringoscopia debe ser combinada con el uso de instrumentos ópticos. Por su parte la citología laríngea puede ser usada para detectar lesiones precoces pero no reemplaza el examen histológico. Lo mismo sucede con la endoscopía de contacto y la endoscopía autofluorescente. La endoscopía compacta ${ }^{46}$ es un término más reciente que combina la autofluorescencia y la endoscopia de contacto.

En Cuba la mayor incidencia y mortalidad en hombres de cáncer de la glotis, cuya evolución clínica permite un diagnóstico más temprano, la estructura de los servicios de salud podrían hacer factible una detección más temprana de la enfermedad. En este sentido sería recomendable evaluar la factibilidad y eficacia de la realización del examen ORL (laringoscopia indirecta) ${ }^{14}$-método no invasivo y que requiere de pocos recursos-, como parte del examen clínico periódico anual, fundamentalmente en hombres de 50 años y más, con historia de tabaquismo y/o alcoholismo, con el fin de pesquisar y tratar tempranamente lesiones asintomáticas. 
En este contexto vale la pena recordar los principios fundamentales y necesarios para la puesta en práctica de un pesquisaje masivo de control del cáncer definidos por Wilson y Youngner: ${ }^{47,48}$

...tratarse de una enfermedad frecuente y conocida con una fase de enfermedad preclínica o período de latencia suficientemente largo, que pueda ser puesta en evidencia por una prueba relativamente sencilla, fácil de realizar y que no entrañe secuelas para el paciente - como es el caso de la laringoscopia indirecta-, que sea una prueba con suficiente y probada validez - medida por su sensibilidad, especificidad y valores predictivos-, que existan pruebas subsiguientes para la confirmación del diagnóstico; la prueba debe ser atractiva y no invasiva facilitando su aceptabilidad por la población beneficiaria a un costo adecuado, y por supuesto que se cuente con un tratamiento oportuno y adecuado, que conlleve a minimizar el detrimento de la calidad de vida del enfermo, todo lo cual garantice su eficacia y efectividad.

\section{REFERENCI AS BI BLI OGRÁFI CAS}

1. Álvarez JJ, Brandáriz JA, García A. Cáncer de laringe. En: Cortés-Funes H, DíazRubio E, García-Conde J, Germá Lluch R, Guillem Porta V, López López JJ, Moreno Nogueira JA, Pérez Manga G, editores. Oncología Clínica. España: Nova Sidonia Oncología (Grupo Aula Médica, S.A.);2000. p.325-42.

2. International Agency for Research on Cancer (IARC). Tobacco habits other than smoking; betel-quid and areca-nut chewing and some related nitrosamines (IARC Monographs on the Evaluation of Carcinogenic Risk to Humans, Vol. 37). Lyon, France: IARCPress; 1985.

3. International Agency for Research on Cancer (IARC). Tobacco smoking (IARC Monographs on the Evaluation of the Carcinogenic Risk of Chemicals to Humans, Vol. 38). Lyon, France: IARCPress; 1986.

4. International Agency for Research on Cancer (IARC). Alcohol Drinking (IARC Monographs on the Evaluation of Carcinogenic Risks to Humans, Vol. 44). Lyon, France: IARCPress; 1988.

5. Stewart BW, Kleihues P. World Cancer Report. Lyon: WHO International Agency for Research on Cancer; 2003.

6. Tuyns AJ, Estève J, Raymond L, Berrino F, Benhamou E, Blanchet F, et al. Cancer of the larynx/hypopharynx, tobacco and alcohol: IARC international case-control study in Turin and Varese (Italy), Zaragoza and Navarra (Spain), Geneva (Switzerland) and Calvados (France). Int J Cancer. 1988;41:483-91.

7. Arbesun O. Síndrome disfónico. Otorrinolaringología. En: Arbesun Ruiz O, editor científico. Temas de Otorrinolaringología [sitio en Internet]. 2006 [citado 2008]. Disponible en: http://www.sld.cu/sitios/otorrino/temas.php?idv=12406

8. J ofre D. Otorrinolaringología. Patología de la laringe [sitio en Internet]. 2006 [citado 2008]. Disponible en: http://escuela.med.puc.cl/paginas/publicaciones/Otorrino/Otorrino_A010.html 
9. Marck PA, Lupin AJ. Cancer of the larynx: the Northern Alberta experience. J Otolaryngol. 1989; 18: 344-9.

10. Yang PC, Thomas DB, Daling J R, Davis S. Differences in the sex ratio of laryngeal cancer incidence rates by anatomic subsite. J Clin Epidemiol. 1989; 42: 755-8.

11. Robin PE, Reid A, Powell DJ, McConkey CC. The incidence of cancer of the larynx. Clin Otolaryngol. 1991;16:198-201.

12. Stephenson WT, Barnes DE, Holmes FF, Norris CW. Gender influences subsite of origin of laryngeal carcinoma. Arch Otolaryngol Head Neck Surg. 1991;117: 774-8.

13. Tuyns AJ . Laryngeal cancer. Cancer Surv. 1994; 19-20: 159-73.

14. Villar MS. Laringología. En: Villar Suárez MS, editor. Otorrinolaringología. 2da ed. La Habana: Editorial Ciencias Médicas; 2004. p.137-61.

15. Ministerio de Salud Pública, Dirección Nacional de Estadísticas, Cuba. Anuario Estadístico de Salud [sitio en Internet]. 2007 [citado 2008]. Disponible en: http://www.sld.cu/servicios/estadisticas/

16. Percy C, Van Holten V, Muir C. ICD-O: International Classification of Diseases for Oncology. 2nd ed. Geneva:WHO; 1976.

17. Gallus S, Bosetti C, Franceschi S, Levi F, Negri E, La Vecchia C. Laryngeal Cancer in Women: Tobacco, Alcohol, Nutritional, and Hormonal Factors. Cancer Epidemiology. Biomarkers \& Prevention. 2003; 12: 5147.

18. Parkin DM, Whelan SL, Ferlay J, Teppo L, Thomas DB, editors. Cancer Incidence in Five Continents. Vol. VIII. Lyon. France: IARCPress; 2002. [IARC Scientific Publications No. 155].

19. Cattaruzza MS, Maisonneuve P, Boyle P. Epidemiology of Laryngeal Cancer. Oral Oncol, Eur J Cancer. 1996; 32B (5):293-305.

20. Peng J, Raverdi N, Ganry O, De La Roche-Saint André G, Dubreuil A, Lorriaux A. Descriptive epidemiology of upper aerodigestive tract cancers in the department of Somme. Bull Cancer. 2000;87(2): 201-6.

21. Soriano J, Galán Ya, Luaces P, Martín A, Arrebola J, Carrillo G. Incidencia en Cuba del cáncer en la tercera edad. Rev Cubana Oncol. 1998;4(2):121-8.

22. Virtaniemi J, Hivikoski P, Kumpulainen E, Johansson R, Pukkala E, Kosma V. Is the subsite distribution of laryngeal cancer related to smoking habits? Acta Oncologica. 2000; 9 (1):77-9.

23. Brugére J, Guénel $P$, Leclerc A, Rodriguez J. Differential effects of tobacco and alcohol in cancer of the larynx, pharynx, and mouth. Cancer. 1986; 57:391-5.

24. Guénel P, Engholm G, Lynge E. Laryngeal cancer in Denmark: a nationwide longitudinal study based on register linkage data. $\mathrm{Br} J$ Industr Med. 1990;47:473-9. 
25. Merletti F, Faggiano F, Boffetta P, Lehmann W, Rombola A, Amasio E, et al. Topographic classification, clinical characteristics, and diagnostic delay of cancer of the larynx/hypopharynx in Torino, I taly. Cancer. 1990;66:1711-6.

26. Silvestri F, Bussani R, Stanta G, Cosatti C, Ferlito A. Supraglottic versus glottic laryngeal cancer: epidemiological and pathological aspects. ORL J Otorhinolaryngol Relat Spec. 1992; 54:43-8.

27. Basut O, Tezel I, Erisen L, Coskun H. Topographic distribution of laryngeal cancer. J Environ Pathol Toxicol Oncol. 1996; 15:231-2.

28. Kurtulmaz SY, Erkal HS, Serin M, Elhan AH, Cakmak A. Squamous cell carcinomas of the head and neck: descriptive analysis of 1293 cases. J Laryngol Otol. 1997; 111:531-5.

29. De Stéfani E, Carzoglio J, Cendán M, Deneo H, Olivera L, Oreggia F. Laryngeal cancer in Uruguay (1958-1981). An epidemiologic study. Cancer. 1985; 55:214-6.

30. De Stéfani E, Correa E, Oreggia F, Leiva J, Rivero S, Fernandez G, et al. Risk Factors for laryngeal cancer. Cancer. 1987; 60:3087-91.

31. Raitiola H. Epidemiology, Clinical Characteristics and Treatment Outcome of Laryngeal Cancer. Academic Dissertation. University of Tampere. Medical School. Finland. (Acta Electronica Universitatis Tamperensis 38) [sitio en Internet]. 2000 [citado 2007]. Disponible en: http://acta.uta.fi/pdf/951-44-4828-6.pdf

32. Lauerma S. Treatment of laryngeal cancer. A study of 638 cases. Acta Otolaryngol Suppl (Stockh). 1967;225: 1-101.

33. De Stéfani E, Boffetta P, Deneo-Pellegrini H, Brennan P, Correa P, Oreggia F, et al. Supraglottic and glottic carcinomas: epidemiologically distinct entities? Int J Cancer. 2004; 112(6): 1065-71.

34. Menvielle G, Luce D, Goldberg P, Bugel I, Leclerc A. Smoking, alcohol drinking and cancer risk for various sites of the larynx and hipopharynx. A case-control study in France. Eur J Cancer Prev. 2004; 13: 165-72.

35. Hashibe M, Boffetta P, Zaridze D, Shangina O, Szeszenia-Dabrowska N, Mates $D$, et al. Contribution of tobacco and alcohol to the highrRates of squamous cell carcinoma of the supraglottis and glottis in Central Europe. Am J Epidemiol. 2007; 165(7): 814-20.

36. Harris J A, Meyers AD, Smith C. Laryngeal cancer in Colorado. Head Neck. 1993; 15: 398-404.

37. Grénman R, Pekkola-Heino K, Kinnala P. The incidence of laryngeal cancer by anatomical site in south-western Finland (letter). Eur Arch Otorhinolaryngol. 1996; 253: 377.

38. León X, Quer M, Agudelo D, López-Pousa A, De Juan M, Diez S, Burgués J. Influence of age on laryngeal carcinoma. Ann Otorhinolaryngol. 1998; 107: 164-9. 
39. Hirvikoski P, Virtaniemi J, Kumpulainen E, J ohansson R, Kosma VM. Supraglottic and glottic carcinomas. clinically and biologically distinct entities? Eur J Cancer. 2002; 38(13): 1717-23.

40. WHO. National Cancer Control Programmes. Policies and managerial guidelines. 2nd ed. [sitio en Internet]. 2002 [citado Ago 2008]. Disponible en: http://www. who.int/cancer/media/en/408.pdf

41. Smith RP, MD Hill-Kayser C. Laryngeal Cancer: The Basics. The Abramson Cancer Center of the University of Pennsylvania [sitio en Internet]. [citado Ago 2008]. Disponible en: Oncolink:

http://www.oncolink.com/types/article. $\mathrm{fmm} ? \mathrm{c}=7 \& \mathrm{~s}=24 \& \mathrm{ss}=185 \& \mathrm{id}=9450$

42. Hoare TJ, Thomson HG, Proops DW. Detection of laryngeal cancer - the case for early specialist assessment. J Royal Society Med. 1993;86:390-2. Disponible en: http://www.pubmedcentral.nih.gov/articlerender.fcgi?artid=1293007

43. Abtahi SM. Evaluating hoarseness in early diagnosis of laryngeal cancer [letter]. J Res Med Sci. 2005; 10 (3): 185. Disponible en:

http://journals.mui.ac.ir/jrms/article/view/105/65

44. Malzahn K, Dreyer T, Glanz H, Arens C. Autofluorescence endoscopy in the diagnosis of early laryngeal cancer and its precursor lesions. The Laryngoscope. 2002; 112(3): 488-49. Disponible en:

http: // cat.inist.fr/ ?aModele $=$ afficheN\&cpsidt $=13538003$

45. Arens C, Dreyer T, Glanz H, Malzahn K. Indirect autofluorescence laryngoscopy in the diagnosis of laryngeal cancer and its precursor lesions. Eur Arch

Otorhinolaryngol. 2004;261(2): 71-6. Disponible en:

http://www.springerlink.com/content/v77dmc06898m1jfa/

46. Olofsson J, Sykehus H. Early detection of laryngeal cancer. German Med Sci [serie en Internet]. 2008 [citado Ago 2008]. Disponible en:

http://www.egms.de/en/meetings/hno2008/08hno62.shtml [German Society of Oto-Rhino-Laryngology, Head and Neck Surgery. 79th Annual Meeting of the German Society of Oto-Rhino-Laryngology, Head and Neck Surgery. Bonn, 30.04.04.05.2008 Düsseldorf, Köln. Doc 08hno62].

47. Miller AB. La detección temprana y el tamizaje en el control del cáncer. Bol Oficina Sanit Panam. 1995; 118(1): 8083.

48. Haehnel P. Dépistage: principes éthiques. Bull Cancer. 200;88(4):389-90.

Recibido: 2 de septiembre de 2008 .

Aprobado: 24 de octubre de 2008. 
Juan J. Lence Anta. Instituto Nacional de Oncología y Radiobiología. 29 y F. EI Vedado. La Habana 10400, Cuba.

Telf. 6981408. E-mail: lence@infomed.sld.cu 